\title{
Application of Optimal HAM for Finding Feedback Control of Optimal Control Problems
}

\author{
H. Saberi Nik ${ }^{1}$ and Stanford Shateyi ${ }^{2}$ \\ ${ }^{1}$ Department of Mathematics, Neyshabur Branch, Islamic Azad University, Neyshabur, Iran \\ ${ }^{2}$ Department of Mathematics, University of Venda, Private Bag X5050, Thohoyandou 0950, South Africa \\ Correspondence should be addressed to Stanford Shateyi; stanford.shateyi@univen.ac.za
}

Received 8 November 2012; Accepted 1 February 2013

Academic Editor: Trung Nguyen Thoi

Copyright (c) 2013 H. Saberi Nik and S. Shateyi. This is an open access article distributed under the Creative Commons Attribution License, which permits unrestricted use, distribution, and reproduction in any medium, provided the original work is properly cited.

\begin{abstract}
An optimal homotopy-analysis approach is described for Hamilton-Jacobi-Bellman equation (HJB) arising in nonlinear optimal control problems. This optimal approach contains at most three convergence-control parameters and is computationally rather efficient. A kind of averaged residual error is defined. By minimizing the averaged residual error, the optimal convergence-control parameters can be obtained. This optimal approach has general meanings and can be used to get fast convergent series solutions of different types of equations with strong nonlinearity. The closed-loop optimal control is obtained using the Bellman dynamic programming. Numerical examples are considered aiming to demonstrate the validity and applicability of the proposed techniques and to compare with the existing results.
\end{abstract}

\section{Introduction}

Many real-world problems in engineering, economics, and biomedicine can be formulated as optimal control problem of the form

$$
\begin{array}{ll}
\text { minimum } & J=h\left(x\left(t_{f}\right), t_{f}\right)+\int_{t_{0}}^{t_{f}} g(x(s), u(s), s) d s, \\
\text { subject to } & \dot{x}(t)=f(x(t), u(t), t), \\
& x\left(t_{0}\right)=x_{0}, \quad x\left(t_{f}\right)=x_{f},
\end{array}
$$

where $u(\cdot)$ is the control function, $x(\cdot)$ is the state function, constant parameters, $x_{0}$ and $x_{f}$ are the initial and the final states, $h(\cdot)$ is the salvage value or scrap value function, $f(\cdot)$ is the vector-valued transition function, $g(\cdot)$ is the integrand function, and $s$ is the dummy variable of integration rather than $t$.

There are various efficient methods such as those reported in [1-3] for the computation of open-loop optimal controls. However, feedback controls are much preferred in many engineering applications. In order to determine the optimal control law, there is an approach using dynamic programming [4]. This approach leads to the HJB equation that is hard to solve in most cases. An excellent literature review on the methods for solving the HJB equation is provided in [5], where a successive Galerkin approximation (SGA) method is also considered. In the SGA, a sequence of generalized HJB equations is solved iteratively to obtain a sequence of approximations approaching the solution of HJB equation. However, the proposed sequence may converge very slowly or even diverge. Another approach is to treat the problem with a measure theory approach [6]. This changes the nonlinear OCP to a linear programming and gives a piecewise constant control law. In the past two decades, the indirect methods have been extensively developed. It is well known that the nonlinear OCP leads to a nonlinear two-point boundary value problem (TPBVP) or a Hamilton-JacobiBellman partial differential equation. Many recent researches have been devoted to solve these two problems.

In particular, we will derive the fundamental first-order partial differential equation obeyed by the optimal value function, known as the HJB equation. This shift in our attention, moreover, will lead us to a different form for the optimal value of the control vector, namely, the feedback or 
closed-loop form of the control. This form of the optimal control typically gives the optimal value of the control vector as a function of the current date, the current state, and the parameters of the control problem. In contrast, the form of the optimal control vector derived via the necessary conditions of optimal control theory is termed open loop and in general gives the optimal value of the control vector as a function of the independent variable time, the parameters, and the initial and/or terminal values of the planning horizon and the state vector. Essentially, the feedback form of the optimal control is a decision rule, for it gives the optimal value of the control for any current period and any admissible state in the current period that may arise. In contrast, the open-loop form of the optimal control is a curve, for it gives the optimal values of the control as the independent variable time varies over the planning horizon. Thus, it is required to solve an $\mathrm{HJB}$ equation by numerical-analyticapproximate method. In [7], the authors used the basic ADM for an example of HJB equation and then finding optimal control signal. In [8-10], standard HPM, piecewise HPM and differential transform method (DTM) were used for finding feedback control of optimal control problems.

One of the semiexact methods for solving linear and nonlinear equation which does not need small/large parameters is homotopy analysis method (HAM), first proposed by Liao in 1992 [10-13]. Since Liao's book [14] for the homotopy analysis method was published in 2003, more and more researchers have been successfully applying this method to various nonlinear problems in science and engineering, such as the viscous flows of non-Newtonian fluids [15], the KdVtype equations [16], and finance problems [17].

The HAM contains a certain auxiliary parameter $c_{0}$, which provides us with a simple way to adjust and control the convergence region and rate of convergence of the series solution. Moreover, by means of the so-called $c_{0}$-curve, it is easy to determine the valid regions of $c_{0}$ to gain a convergent series solution. Thus, through HAM, explicit analytic solutions of nonlinear problems are possible.

However, as illustrated in [18], the usual HAM has only one convergence-control parameter $c_{0}$, and it is a pity that curves for convergence-control parameter (i.e., $c_{0}$-curves) cannot tell us which value of $c_{0} \in \mathbb{R}$ gives the fastest convergent series. Recently, to overcome this shortcoming, Liao [18] proposed an optimal HAM with more than one convergence-control parameter. In this optimal method, Liao introduced the so-called averaged residual error to get the optimal convergence-control parameters efficiently. In general, the optimal HAM can greatly modify the convergence of homotopy-series solution.

The results obtained in this paper show that the solutions given by the optimal HAM give much better approximations and convergence much faster than those given by the usual HAM. In this paper, we employed a new proposal of Liao [18, 19], namely, optimal homotopy-analysis approach to solve an HJB equation obtained from (2)-(4). We use here special deformation functions, which are determined completely by only one characteristic parameter $\left|c_{1}\right|<1$ and $\left|c_{2}\right|<$ 1 , respectively. In this way, there exist at most only three convergence-control parameters $c_{0}, c_{1}$, and $c_{2}$ at any order of approximations.

\section{Nonlinear Time-Variant HJB Equation}

Consider a process described by the following system of nonlinear differential equations, which is called the equation of motion, on a fixed interval $\left[t_{0}, t_{f}\right]$

$$
\dot{x}(t)=f(x(t), u(t), t),
$$

where $x \in \mathbb{R}^{n}$ is the state vector. Let $U(\cdot) \in \mathbb{R}^{n}$ be a closed set. A piecewise continuous function $u:\left[t_{0}, t_{f}\right] \rightarrow \mathbb{R}^{n}$ is said to be an admissible control if $u(t) \in U(\cdot)$. Let $\mathcal{U}$ be the class of such admissible controls. The function $f$ is a real-valued continuously differentiable function and has continuous first partial derivative with respect to $x$. The initial condition for (2) is

$$
x\left(t_{0}\right)=x_{0} .
$$

Along with this controlled process, we have a cost functional of the form

$$
J\left(x_{0}, u\right)=\Phi\left(x\left(t_{f}\right), t_{f}\right)+\int_{t_{0}}^{t_{f}} L(x(\tau), u(\tau), \tau) d \tau .
$$

Here, $L(x(\tau), u(\tau), \tau)$ is the running cost, and $\Phi\left(x\left(t_{f}\right), t_{f}\right)$ is the terminal cost. This cost functional depends on the initial position $\left(x_{0}, t_{0}\right)$ and the choice of control $u(\cdot)$. The optimization problem is therefore to minimize $J\left(x_{0}, u\right)$, for each $\left(x_{0}, u\right)$, over all controls $u(t) \in U(\cdot)$. The pair $\left(x_{0}, u\right)$ which achieves this minimum is called an optimal control. In fact, the optimization problem with performance index as in (4) is called a Bolza problem.

In dynamic programming, the minimum value of the performance index is considered as a function of this initial point. This function is called the value function. Whenever the value function is differentiable, it satisfies a nonlinear first-order hyperbolic partial differential equation called the partial differential equation of dynamic programming. This equation is used for constructing a nonlinear optimal feedback control law. If we consider a family of optimization problems with differential initial condition $(x, t)$, we consider the dependence of the value of these optimization problems on their initial conditions. Thus, a value function is defined by

$$
\begin{aligned}
V(x, t) & =J\left(x, u^{*}\right) \\
& =\min _{u \in \mathscr{U}}\left\{\Phi\left(x\left(t_{f}\right), t_{f}\right)+\int_{t}^{t_{f}} L(x(\tau), u(\tau), \tau) d \tau\right\} .
\end{aligned}
$$

Theorem 1. Let $(x, t)$ be any interior point of the set $\{(x, t) \in$ $\left.\mathbb{R}^{n+1} \mid \mathcal{U} \neq 0\right\}$ at which the function $V(x, t)$ is differentiable. Function $V(x, t)$ satisfies the partial differential inequality

$$
\frac{\partial V}{\partial t}+\frac{\partial V}{\partial x} f(x, u, t)+L(x, u, t) \geq 0,
$$


for all $u \in \mathcal{U}$. If there is an optimal control $u^{*} \in \mathcal{U}$, then the partial differential equation

$$
\frac{\partial V}{\partial t}+\min _{u \in U}\left\{\frac{\partial V}{\partial x} f(x, u, t)+L(x, u, t)\right\}=0
$$

is satisfied [20].

This nonlinear time-variant differential equation is called "HJB equation." We have the following boundary condition:

$$
J^{*}\left(x\left(t_{f}\right), t_{f}\right)=V\left(x\left(t_{f}\right), t_{f}\right)=\Phi\left(x\left(t_{f}\right), t_{f}\right),
$$

and by introducing the Hamiltonian function

$$
H\left(x, u, V_{x}, t\right)=\frac{\partial V}{\partial x} f(x, u, t)+L(x, u, t),
$$

we have

$$
H\left(x, u^{*}, V_{x}, t\right)=\min _{u \in U} H\left(x, u, V_{x}, t\right) .
$$

Therefore, by substitution of Hamiltonian function (10) in (7), we have

$$
\frac{\partial V}{\partial t}+H\left(x, u^{*}, V_{x}, t\right)=0
$$

\section{Basic Idea of Optimal Homotopy Analysis Method}

In this section, we give a brief description of optimal homotopy-analysis approaches in general cases. For a general nonlinear differential/integral equation as follows:

$$
N(U(r, t))=0
$$

where $U(r, t)$ is an unknown function, $r$ and $t$ denote, respectively, spatial and temporal independent variables, we can always choose a proper initial guess $U_{0}(r, t)$ and a proper auxiliary linear operator $L$ to construct the so-called zerothorder deformation equation

$$
\begin{aligned}
& {\left[1-B_{n^{\prime}}(q ; b)\right] L\left[\phi(r, t ; q)-U_{0}(r, t)\right]} \\
& \quad=c_{0} A_{m^{\prime}}(q, a) N[\phi(r, t ; q)], \quad q \in[0,1],
\end{aligned}
$$

where $A_{m^{\prime}}(q, a)$ is a deformation function with $m^{\prime}\left(m^{\prime} \geq\right.$ $0)$ unknown convergence-control parameters denoted by $c_{1}, c_{2}, \ldots, c_{m}^{\prime}$, and $B_{n^{\prime}}(q, b)$ is a deformation function with $n^{\prime}\left(n^{\prime} \geq 0\right)$ unknown convergence-control parameters denoted by $c_{m^{\prime}+1}, c_{m^{\prime}+2}, \ldots, c_{m^{\prime} n^{\prime}}$, respectively. Here,

$$
a=\left\{c_{1}, c_{2}, \ldots, c_{m^{\prime}}\right\}, \quad b=\left\{c_{m^{\prime}+1}, c_{m^{\prime}+2}, \ldots, c_{m^{\prime}+n^{\prime}}\right\} .
$$

Put $l=m^{\prime}+n^{\prime}$. Then, we have $l+1$ unknown convergencecontrol parameters $c_{0}, c_{1}, c_{2}, \ldots, c_{l}$. Assuming that the initial guess $U_{0}(r, t)$, the auxiliary linear operator $L$, and the $l+1$ convergence-control parameters $c_{1}, c_{2}, \ldots, c_{l}$ are so properly chosen that the Taylor series

$$
\phi(r, t ; q)=U_{0}(r, t)+\sum_{n=1}^{+\infty} U_{n}(r, t) q^{n}
$$

converges at $q=1$, we have the homotopy-series solution

$$
U(r, t)=U_{0}(r, t)+\sum_{n=1}^{+\infty} U_{n}(r, t) .
$$

Substituting the series (15) into the zeroth-order deformation equation (13) and then equating the coefficients of the like power of the embedding parameter $q$, we have the high-order deformation equation. Consider

$$
L\left[U_{m}(r, t)-\sum_{k=1}^{m-1} \sigma_{m-k}(b) U_{k}(r, t)\right]=c_{0} \sum_{k=0}^{m-1} \mu_{m-k}(a) \delta_{k}(r, t),
$$

where

$$
\delta_{k}(r, t)=\left.\left(\frac{1}{k !} \frac{\partial^{k}}{\partial q^{k}} N\left[\sum_{n=1}^{+\infty} U_{n}(r, t) q^{n}\right]\right)\right|_{q=0},
$$

and $\mu_{k}(a)$ and $\sigma_{k}(b)$ are coefficients of the Taylor series

$$
A_{m^{\prime}}(q ; a)=\sum_{k=1}^{+\infty} \mu_{k}(a) q^{k}, \quad B_{n^{\prime}}(q ; b)=\sum_{k=1}^{+\infty} \sigma_{k}(b) q^{k} .
$$

Let $A_{m^{\prime}}(q ; a), B_{n^{\prime}}(q ; b)$ be complex functions analytic in the region $|q| \leq 1$, which satisfy

$$
A_{m^{\prime}}(0 ; a)=B_{n^{\prime}}(0 ; a)=0, \quad A_{m^{\prime}}(1 ; a)=B_{n^{\prime}}(1 ; a)=1 \text {. }
$$

The special solution $U_{m}^{*}(r, t)$ of $(17)$ is given by

$$
U_{m}^{*}(r, t)=\sum_{k=1}^{m-1} \sigma_{m-k}(b) U_{k}(r, t)+c_{0} \sum_{k=1}^{m-1} \mu_{m-k}(a) S_{k}(r, t),
$$

where

$$
S_{k}(r, t)=L^{-1}\left[\delta_{k}(r, t)\right],
$$

and $L^{-1}$ is the inverse operator of $L$.

Now, we define a kind of averaged residual error [18]

$$
E_{m}=\frac{1}{k} \sum_{j=0}^{k}\left[N\left(\sum_{i=0}^{m} U_{i}(j \Delta t)\right)\right]^{2} .
$$

The value of $k$ is depends to problem, for Examples 2 and 3, we have $\Delta t=1 / 10, k=10$, and $\Delta t=1 / 20, k=20$, respectively.

Note that $E_{m}$ contains $l+1$ unknown convergencecontrol parameters $c_{0}, c_{1}, \ldots, c_{l}$, whose optimal values are given by the minimum of $E_{m}$, corresponding to a set of $l+1$ nonlinear algebraic equations

$$
\frac{\partial E_{m}}{\partial c_{j}}=0, \quad j=0,1, \ldots, l .
$$

So, the above approach is called the $l+1$-parameter optimal homotopy-analysis approach. In general, the above-mentioned optimal homotopy-analysis approaches can greatly modify the convergence of homotopy-series solution. And the optimal homotopy-analysis approaches with one or two unknown convergence-control parameters are strongly suggested; an optimal approach with too many unknown convergence-control parameters is not efficient computationally. 


\section{Application}

To illustrate the effectiveness of the optimal homotopy analysis method, we will consider two examples. Comparisons are made between the exact solution and optimal homotopyanalysis approaches.

Example 2. Consider the following purely mathematical optimal control problem:

$$
\dot{x}=x(t)+u(t) .
$$

Suppose that we consider the following cost function for this system:

$$
J=x^{2}\left(t_{f}\right)+\int_{0}^{t_{f}} u^{2}(t) d t .
$$

The Hamiltonian function will be

$$
H\left(x, u, V_{x}, t\right)=u^{2}(t)+V_{x}(x, t)[x+u] .
$$

Since the Hamiltonian, which as you know is the right-handside expression to be minimum, is a convex function of the state and control variables, a solution of the necessary conditions is a solution of the optimal control. Thus, the necessary and sufficient condition for the above minimization problem in the HJB equation is given by

$$
\frac{\partial H}{\partial u}=2 u(t)+V_{x}(x, t)=0,
$$

which is easily solved to get

$$
u^{*}(t)=-\frac{1}{2} V_{x}(x, t) .
$$

Because $\partial^{2} H / \partial u^{2}=2>0, u^{*}$ is a minimum and acceptable. Now, by substitution of $u^{*}$ in HJB equation, we have the following:

$$
\begin{gathered}
-V_{t}=-\frac{1}{4} V_{x}^{2}+x V_{x}, \\
V\left(x\left(t_{f}\right), t_{f}\right)=x^{2}\left(t_{f}\right) .
\end{gathered}
$$

In Chapter 19 of [21], the authors obtained the solution of the above HJB equation in the form

$$
V(x, t)=\frac{2 x^{2}}{1+e^{2\left(t-t_{f}\right)}}, \quad u^{*}(t)=-\frac{2 x}{1+e^{2\left(t-t_{f}\right)}},
$$

for simplicity, let $u^{*}(x, t)=k(t) x(t)$, where

$$
k(t)=\frac{-2}{1+e^{2\left(t-t_{f}\right)}} .
$$

To solve (30) by means of optimal homotopy analysis method, we choose the initial approximation, final time, and auxiliary linear operator

$$
\begin{gathered}
V_{0}(x, t)=x^{2}, \quad t_{f}=1, \\
L[\phi(x, t ; q)]=\frac{\partial \phi(x, t ; q)}{\partial t} .
\end{gathered}
$$

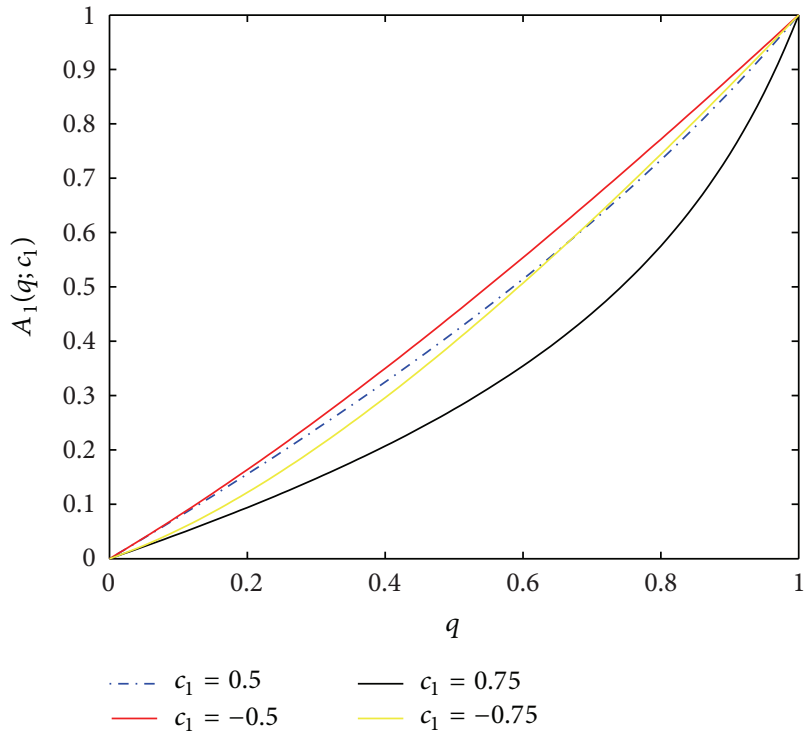

FIgURE 1: Deformation function $A_{1}\left(q ; c_{1}\right)$ defined by (34) and (35).

There are an infinite number of deformation functions satisfying the properties (19). For the sake of computation efficiency, we use here the following one-parameter deformation functions:

$$
A_{1}\left(q ; c_{1}\right)=\sum_{m=1}^{+\infty} \mu_{m}\left(c_{1}\right) q^{m}, \quad B_{1}\left(q ; c_{2}\right)=\sum_{m=1}^{+\infty} \sigma_{m}\left(c_{2}\right) q^{m}
$$

where $\left|c_{1}\right|<1$ and $\left|c_{2}\right|<1$ are constants, called the convergence-control parameter, and

$$
\begin{aligned}
& \mu_{m}\left(c_{1}\right)=\left(1-c_{1}\right) c_{1}^{m-1}, \quad m \geq 1, \\
& \sigma_{m}\left(c_{2}\right)=\left(1-c_{2}\right) c_{2}^{m-1}, \quad m \geq 1 .
\end{aligned}
$$

The different values of $c_{1}$ give different paths of $A_{1}\left(q ; c_{1}\right)$, as shown in Figure 1.

Let $q \in[0,1]$ denote the embedding parameter, $c_{0} \neq 0$ an auxiliary parameter, called the convergence-control parameter, and $\phi(x, t ; q)$ a kind of continuous mapping of $V(x, t)$, respectively. Obviously, when $q=0$ and $q=1$, it holds

$$
\phi(x, t ; 0)=V_{0}(x, t), \quad \phi(x, t ; 1)=V(x, t),
$$

respectively. Thus, as $q$ increases from 0 to 1 , the solutions $\phi(x, t ; q)$ vary from the initial guesses $V_{0}(x, t)$ to the solutions $V(x, t)$. We construct the zeroth-order deformation equation:

$$
\begin{aligned}
& {\left[1-B_{1}\left(q ; c_{2}\right)\right] L\left[\phi(x, t ; q)-V_{0}(x, t)\right]} \\
& \quad=c_{0} A_{1}\left(q, c_{1}\right) N[\phi(x, t ; q)], \quad q \in[0,1],
\end{aligned}
$$

subject to the boundary condition

$$
\phi(x(1), 1 ; 0)=x^{2}(1) .
$$


As mentioned, we have the $m$ th-order deformation equation

$$
\begin{gathered}
L\left[V_{m}(x, t)-\sum_{k=1}^{m-1} \sigma_{m-k}\left(c_{2}\right) \mathrm{V}_{k}(x, t)\right] \\
=c_{0} \sum_{k=0}^{m-1} \mu_{m-k}\left(c_{1}\right) \delta_{k}(x, t),
\end{gathered}
$$

where

$$
\delta_{k}(x, t)=\frac{\partial V_{k}}{\partial t}-\frac{1}{4} \sum_{j=0}^{k}\left(\frac{\partial V_{j}}{\partial x}\right)\left(\frac{\partial V_{k-j}}{\partial x}\right)+x \frac{\partial V_{k}}{\partial x}
$$

and the coefficients $\mu_{k}\left(c_{1}\right)$ and $\sigma_{k}\left(c_{2}\right)$ are defined by (35). Let $V^{*}(x, t)$ denote a special solution of (39) and $L^{-1}$ the inverse operator of $L$, respectively. We have

$$
\begin{aligned}
V_{m}^{*}(x, t)= & \sum_{k=1}^{m-1} \sigma_{m-k}\left(c_{2}\right) V_{k}(x, t) \\
& +c_{0} \sum_{k=0}^{m-1} \mu_{m-k}\left(c_{1}\right) L^{-1}\left[\delta_{k}(x, t)\right] .
\end{aligned}
$$

We now successively obtain

$$
\begin{aligned}
V_{1}(x, t)= & -c_{0}\left(-1+c_{1}\right)(t-1) x^{2}, \\
V_{2}(x, t)= & c_{0} t x^{2}-c_{0} x^{2}-c_{0} c_{2} t x^{2}+c_{0} c_{2} x^{2} \\
& +c_{0} c_{2} c_{1} t x^{2}-c_{0} c_{2} c_{1} x^{2}-c_{0} c_{1}^{2} t x^{2} \\
& +c_{0} c_{1}^{2} x^{2}+c_{0}^{2} t x^{2}-c_{0}^{2} x^{2}-2 c_{0}^{2} c_{1} t x_{2} \\
& +2 c_{0}^{2} c_{1} x^{2}+c_{0}^{2} c_{1}^{2} t x^{2}-c_{0}^{2} c_{1}^{2} x^{2}, \\
V_{3}(x, t)= & c_{0} x^{2} t-c_{0} x^{2}-c_{0} c_{2} x^{2} t+c_{0} x^{2} c_{2} \\
& +c_{0} x^{2} c_{2} c_{1} t-c_{0} x^{2} c_{2} c_{1}-c_{0} x^{2} c_{1}^{2} t \\
& +c_{0} x^{2} c_{1}^{2}+c_{0}^{2} x^{2} t-c_{0}^{2} x^{2}-2 c_{0}^{2} x^{2} c_{1} t \\
& +2 c_{0}^{2} x^{2} c_{1}+c_{0}^{2} x^{2} c_{1}^{2} t-c_{0}^{2} x^{2} c_{1}^{2},
\end{aligned}
$$

It should be emphasized that $V_{m}(x, t)$ contains at most three unknown convergence-control parameters $c_{0}, c_{1}$, and $c_{2}$, which determine the convergence region and rate of the homotopy-series solution. Obviously, if the convergencecontrol parameters $c_{0}, c_{1}$, and $c_{2}$ are properly chosen, the homotopy-series solution may converge fast. So, we should find out the good enough values of $c_{0}, c_{1}$, and $c_{2}$, so that the homotopy-series solution converges fast enough.

4.1. Comparisons of Different Approaches for Example 2. In this section, we will give optimal homotopy-analysis approaches with different numbers of unknown convergencecontrol parameters and compare them in detail.

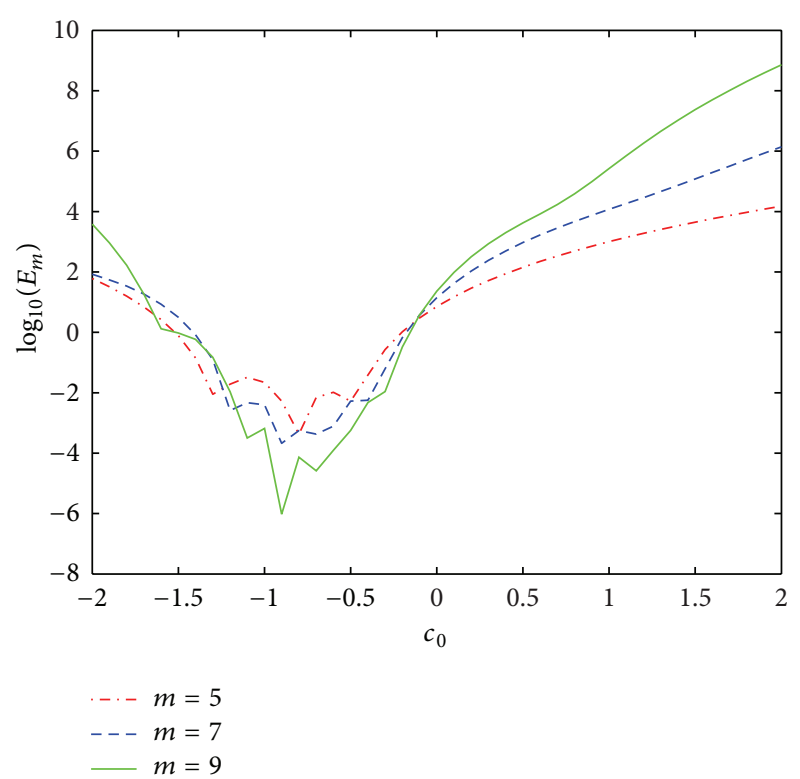

FIgURE 2: Averaged residual error $E_{m}$ versus $c_{0}$ in case of $c_{1}=c_{2}=0$.

TABLE 1: Comparison of averaged residual error given by different $c_{0}$ in case of $c_{1}=c_{2}=0$ and $x=1$.

\begin{tabular}{lcc}
\hline$m$ & Optimal value of $c_{0}$ & Minimum value of $E_{m}$ \\
\hline 5 & -0.8187 & $1.7876 e-004$ \\
7 & -0.8193 & $1.1659 e-004$ \\
9 & -0.8296 & $4.2456 e-006$ \\
\hline
\end{tabular}

4.1.1. Optimal $c_{0}$ in Case of $c_{1}=c_{2}=0$. In this case, only one convergence-control parameter $c_{0}$ is unknown. For given order of approximation $m$, the optimal value of $c_{0}$ is given by the minimum of $E_{m}$, corresponding to a nonlinear algebraic equation $d E_{m} / d c_{0}=0$.

The curves of $E_{m}$ versus $c_{0}$ at a different order of approximation $m=5,7$, and 9 are shown in Figure 2. In case of $c_{1}=c_{2}=0$, there is only one unknown convergence-control parameter $c_{0}$; thus the optimal value of $c_{0}$ is determined by the minimum of $E_{9}$, corresponding to the nonlinear algebraic equation $d E_{9} / d c_{0}=0$. According to Table 1 , the value minimum of $E_{9}$ is obtained at -0.8296 . It should be noted that, when we fixed $c_{0}=-1$ and $c_{1}=c_{2}=0$, automatically the problem turns into the so-called homotopy perturbation method (HPM). So, the HPM is only a special case of the HAM when $c_{0}=-1$.

In case of $c_{1}=c_{2}=0$, one has the plain deformation functions $A_{1}\left(q, c_{1}\right)=B_{1}\left(q, c_{2}\right)=q$, which is exactly the same as that used in traditional HAM.

4.1.2. Optimal $c_{1}=c_{2}$ in Case of $c_{0}=-1$. In this case, $E_{9}$ has the value minimum of $2.8203 \times 10^{-6}$ at the optimal point $c_{1}=$ $c_{2}=0.2458$. This illustrates that the second one-parameter optimal homotopy-analysis approach is as good as the first one mentioned in Section 4.1.1. (See Table 2).

4.1.3. Optimal $c_{0}$ in Case of $c_{1}=c_{2}$. In this case, we obtain the unknown convergence-control parameters $c_{0}$ and $c_{1}$ in case of 
TABLE 2: Comparison of averaged residual error given by different $c_{1}=c_{2}$ in case of $c_{0}=-1$ and $x=1$.

\begin{tabular}{ccc}
\hline$m$ & Optimal value of $c_{1}=c_{1}$ & Minimum value of $E_{m}$ \\
\hline 5 & $c_{1}=c_{2}=0.3971$ & $2.2134 e-004$ \\
7 & $c_{1}=c_{2}=0.3689$ & $1.3731 e-004$ \\
9 & $c_{1}=c_{2}=0.2458$ & $2.8203 e-006$ \\
\hline
\end{tabular}

TABLE 3: Comparison of averaged residual error given by different $c_{0}$ in case of $c_{1}=c_{2}$.

\begin{tabular}{lcc}
\hline$m$ & Optimal value of $c_{0}$ and $c_{1}=c_{2}$ & Minimum value of $E_{m}$ \\
\hline 5 & $c_{0}=-0.9238$ and $c_{1}=c_{2}=0.1001$ & $1.0893 e-004$ \\
7 & $c_{0}=-0.9997$ and $c_{1}=c_{2}=0.1003$ & $1.0425 e-005$ \\
9 & $c_{0}=-1.0012$ and $c_{1}=c_{2}=0.0398$ & $1.4099 e-006$ \\
\hline
\end{tabular}

$c_{2}=c_{1}$. The corresponding residual error $E_{9}$ is now a function of both $c_{0}$ and $c_{1}$, which has the value minimum of $1.4099 \times$ $10^{-6}$ at the optimal point $c_{0}=-1.0012, c_{1}=c_{2}=0.0398$. (See Table 3).

4.1.4. Optimal $c_{0}, c_{1}$, and $c_{2}$ in Case of $c_{1} \neq c_{2}$. Let us consider the optimal approach with the three unknown convergencecontrol parameters. The corresponding $E_{9}$ is now a function of $c_{0}, c_{1}$, and $c_{2}$, which has the minimum $1.7716 \times 10^{-5}$ at the optimal values $c_{0}=-0.6932, c_{1}=-0.2440$, and $c_{2}=-0.1039$. The corresponding homotopy-series solution converges much faster than homotopy-series solution in case of $c_{0}=-1$ and $c_{1}=c_{2}=0$, as shown in Table 4 .

Based on the above calculations for this equation, we found that all optimal homotopy-analysis approaches can give much better approximations, which converge much faster than those without optimal convergence-control parameters in case of $c_{0}=-1$ and $c_{1}=c_{2}=0$, as shown in Tables 1-4.

The absolute error of the 20th-order approximate solution and analytic solution (31) with $c_{0}=-1, c_{1}=c_{2}=0$, and $c_{0}=-0.8296, c_{1}=c_{2}=0$ is shown in Figures 3 and 4, respectively. Comparison of the analytic solution (31) with the optimal homotopy analysis method solution by $c_{0}=-0.8296$ and $c_{1}=c_{2}=0$ is given in Figure 5. The absolute errors $\left|k_{\text {analytic }}-k_{\text {approximate }}\right|$ have been calculated for different cases of $c_{0}, c_{1}$, and $c_{2}$ in Table 5 .

Example 3. Consider a single-input scalar system as follows:

$$
\begin{gathered}
\dot{x}=-2 x(t)+u(t) \\
J=\frac{1}{2} x^{2}(1)+\frac{1}{2} \int_{0}^{1}\left(x^{2}(t)+u^{2}(t)\right) d t .
\end{gathered}
$$

The corresponding Hamiltonian function will be

$$
\begin{aligned}
H\left(x, u, V_{x}, t\right)= & \frac{1}{2} x^{2}(t)+\frac{1}{2} u^{2}(t) \\
& +\frac{\partial V(x, t)}{\partial x}[-2 x(t)+u(t)] .
\end{aligned}
$$

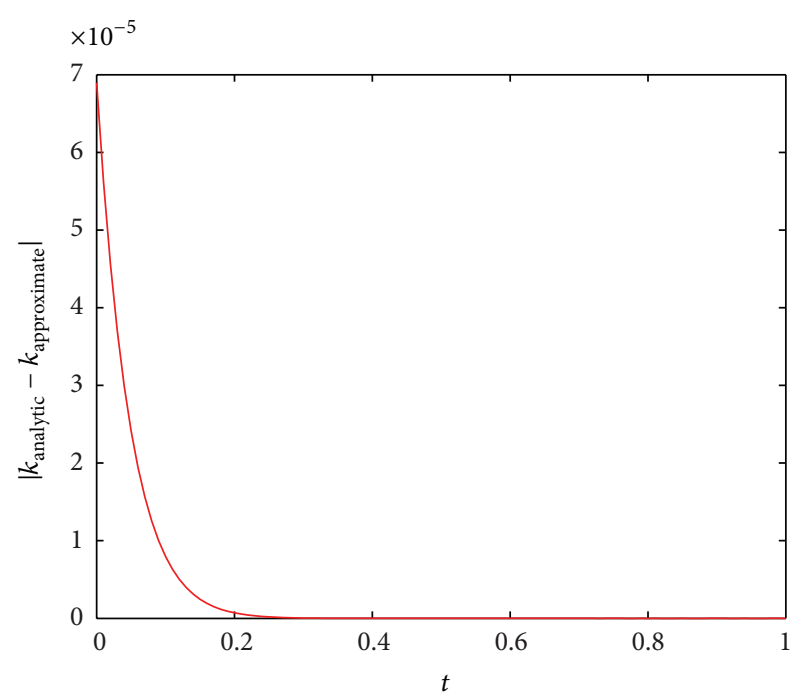

FIGURE 3: $\left|k_{\text {analytic }}-k_{\text {approximate }}\right|(m=20)$, with $c_{0}=-1$ and $c_{1}=c_{2}=$ 0.

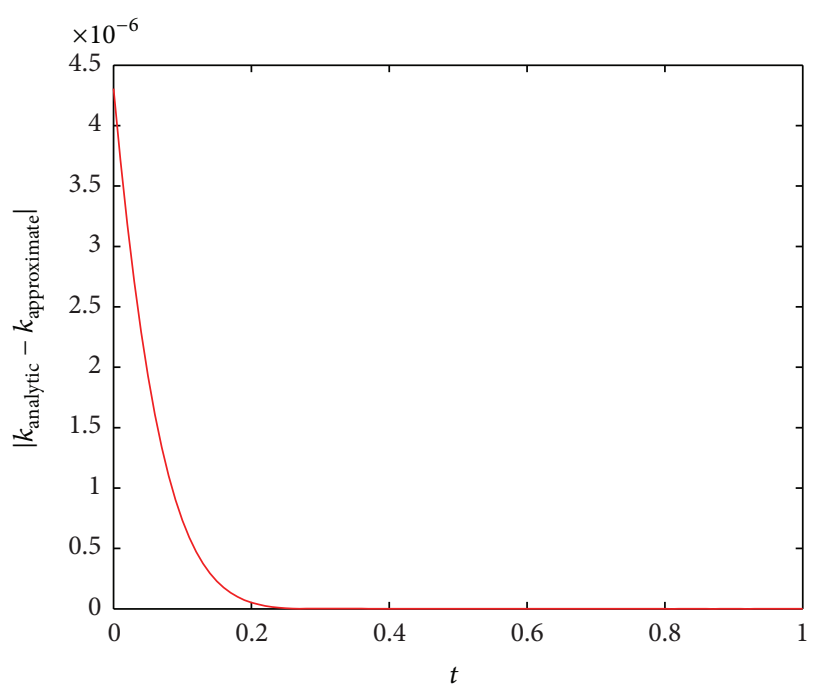

FIGURE 4: $\left|k_{\text {analytic }}-k_{\text {approximate }}\right|(m=20)$, with $c_{0}=-0.8296$ and $c_{1}=c_{2}=0$.

For finding $u^{*}$, we have

$$
\frac{\partial H}{\partial u}=u(t)+\frac{\partial V}{\partial x}=0
$$

Therefore, we obtain

$$
u^{*}(t)=-\frac{\partial V}{\partial x}
$$


TABLE 4: Comparison of averaged residual error given by different approaches.

\begin{tabular}{lccc}
\hline$m$ & $c_{0}=-0.6932, c_{1}=-0.2440$, and $c_{2}=-0.1039$ & $c_{0}=-1, c_{1}=c_{2}=0$ & $c_{0}=-1.0012, c_{1}=c_{2}=0.0398$ \\
\hline 5 & $1.4 e-004$ & $1.4357 e-003$ & $1.0012 e-004$ \\
7 & $4.27026 e-004$ & $4.0327 e-004$ & $1.3265 e-004$ \\
9 & $1.7716 e-005$ & $6.5798 e-005$ & $1.4099 e-006$ \\
\hline
\end{tabular}

TABLE 5: The absolute error of approximate solution and exact solution of $k(t)$ by 20th-order approximate.

\begin{tabular}{lcccc}
\hline$t$ & $c_{0}=-0.8296, c_{1}=c_{2}=0$ & $c_{0}=-1, c_{1}=c_{2}=0$ & $c_{0}=-1.0012, c_{1}=c_{2}=0.0398$ & $c_{0}=-0.6932, c_{1}=-0.2440$, and $c_{2}=-0.1039$ \\
\hline 0 & $2.3195 e-006$ & $6.8965 e-005$ & $3.0012 e-006$ & $8.5935 e-006$ \\
0.2 & $1.2977 e-008$ & $7.0978 e-007$ & $2.3265 e-008$ & $1.0202 e-007$ \\
0.4 & $2.2350 e-010$ & $1.8553 e-009$ & $3.4065 e-010$ & $1.1207 e-009$ \\
0.6 & $4.3885 e-013$ & $4.0035 e-013$ & $1.0039 e-013$ & $1.1423 e-013$ \\
0.8 & $1.9864 e-016$ & 0 & 0 & 0 \\
1.0 & 0 & $3.3307 e-016$ & 0 & 0 \\
\hline
\end{tabular}

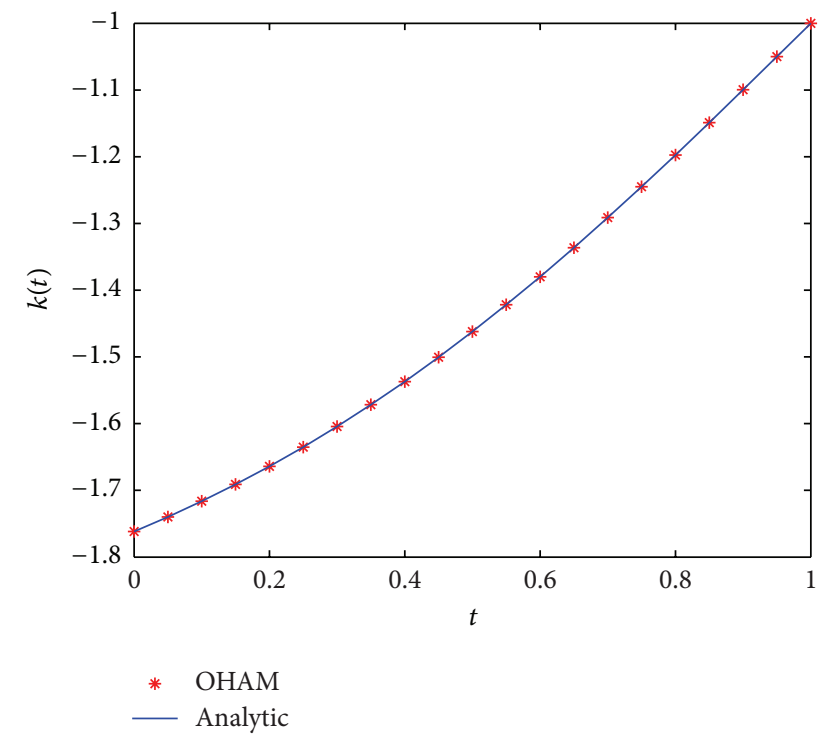

Figure 5: Comparison of the analytic solution (31) with the approximate solution of $k(t)(m=20)$, by $c_{0}=-0.8296$ and $c_{1}=$ $c_{2}=0$.

Because $\partial^{2} H / \partial u^{2}=1>0, u^{*}$ is a minimum and acceptable. Now, by substitution of $u^{*}$ in HJB equation, we have the following:

$$
\begin{aligned}
&-\frac{\partial V}{\partial t}= \frac{1}{2} x^{2}(t)+\frac{1}{2}\left(-\frac{\partial V(x, t)}{\partial x}\right)^{2} \\
&+ \frac{\partial V(x, t)}{\partial x}\left[-2 x(t)-\frac{\partial V(x, t)}{\partial x}\right], \\
& V(x(1), 1)=\frac{1}{2} x^{2}(1)
\end{aligned}
$$

or

$$
\begin{gathered}
\frac{\partial V}{\partial t}=-\frac{1}{2} x^{2}(t)+\frac{1}{2}\left(\frac{\partial V(x, t)}{\partial x}\right)^{2}+2 x \frac{\partial V(x, t)}{\partial x}, \\
V(x(1), 1)=\frac{1}{2} x^{2}(1) .
\end{gathered}
$$

Note that, by (29), the optimal control law is $u^{*}(t)=$ $-k(t) x(t)=f(t) x(t)$, where the exact solution of $k(t)$ is [2]

$$
k(t)=\frac{\sqrt{5} \cosh \sqrt{5}(1-t)-\sinh \sqrt{5}(1-t)}{\sqrt{5} \cosh \sqrt{5}(1-t)+3 \sinh \sqrt{5}(1-t)} .
$$

To solve (48) by means of optimal homotopy analysis method, we choose the initial approximation and auxiliary linear operator

$$
\begin{gathered}
V_{0}(x, t)=\frac{1}{2} x^{2}, \\
L[\phi(x, t ; q)]=\frac{\partial \phi(x, t ; q)}{\partial t} .
\end{gathered}
$$

We construct the zeroth-order deformation equation

$$
\begin{aligned}
& {\left[1-B_{1}\left(q ; c_{2}\right)\right] L\left[\phi(x, t ; q)-V_{0}(x, t)\right]} \\
& \quad=c_{0} A_{1}\left(q, c_{1}\right) N[\phi(x, t ; q)], \quad q \in[0,1],
\end{aligned}
$$

subject to the boundary condition

$$
\phi(x(1), 1 ; 0)=\frac{1}{2} x^{2}(1) .
$$

As mentioned, we have the $m$ th-order deformation equation

$$
\begin{gathered}
L\left[V_{m}(x, t)-\sum_{k=1}^{m-1} \sigma_{m-k}\left(c_{2}\right) V_{k}(x, t)\right] \\
=c_{0} \sum_{k=0}^{m-1} \mu_{m-k}\left(c_{1}\right) \delta_{k}(x, t),
\end{gathered}
$$


TABLE 6: Comparison of averaged residual error given by different $c_{0}$ in case of $c_{1}=c_{2}=0$.

\begin{tabular}{ccc}
\hline$m$ & Optimal value of $c_{0}$ & Minimum value of $E_{m}$ \\
\hline 5 & -0.42943 & $2.9072 e-004$ \\
7 & -0.49327 & $2.9543 e-006$ \\
9 & -0.44032 & $1.3871 e-007$ \\
\hline
\end{tabular}

where

$$
\delta_{k}(x, t)=\frac{\partial V_{k}}{\partial t}-\frac{1}{2} \sum_{j=0}^{k}\left(\frac{\partial V_{j}}{\partial x}\right)\left(\frac{\partial V_{k-j}}{\partial x}\right)-2 x \frac{\partial V_{k}}{\partial x}+\frac{1}{2} x^{2}
$$

and the coefficients $\mu_{k}\left(c_{1}\right)$ and $\sigma_{k}\left(c_{2}\right)$ are defined by (35). Let $V^{*}(x, t)$ denote a special solution of $(53)$ and $L^{-1}$ the inverse operator of $L$, respectively. We have

$$
\begin{aligned}
V_{m}^{*}(x, t)= & \sum_{k=1}^{m-1} \sigma_{m-k}\left(c_{2}\right) V_{k}(x, t) \\
& +c_{0} \sum_{k=0}^{m-1} \mu_{m-k}\left(c_{1}\right) L^{-1}\left[\delta_{k}(x, t)\right] .
\end{aligned}
$$

We now successively obtain

$$
\begin{aligned}
V_{1}(x, t)= & 2 c_{0}\left(-1+c_{1}\right)(t-1) x^{2} \\
V_{2}(x, t)= & -2 c_{0} t x^{2}+2 c_{0} x^{2}+2 c_{0} c_{2} t x^{2} \\
& -2 c_{0} c_{2} x^{2}-2 c_{0} c_{2} c_{1} t x^{2}+2 c_{0} c_{2} c_{1} x^{2} \\
& +6 c_{0} t^{2} x^{2}+8 c_{0}^{2} x^{2}-12 c_{0}^{2} c_{1} t^{2} x^{2} \\
& -16 c_{0}^{2} c_{1} x^{2}+6 c_{0}^{2} c_{1}^{2} t^{2} x^{2} \\
& +8 c_{0}^{2} c_{1}^{2} x^{2}+2 c_{0} c_{1}^{2} t x^{2}-2 c_{0} c_{1}^{2} x^{2} \\
& -14 c_{0}^{2} t x^{2}+28 c_{0}^{2} c_{1} t x^{2}-14 c_{0}^{2} c_{1}^{2} t x^{2},
\end{aligned}
$$

4.2. Comparisons of Different Approaches for Example 3. In this section, we will give optimal homotopy-analysis approaches with different numbers of unknown convergencecontrol parameters and compare them in detail.

4.2.1. Optimal $c_{0}$ in Case of $c_{1}=c_{2}=0$. In this case, only one convergence-control parameter $c_{0}$ is unknown. We solved this example by standard HAM, and we investigated the influence of $c_{0}$ by means of the so-called $c_{0}$-curves. As pointed out by Liao [14], the valid region of $c_{0}$ is a horizontal line segment. So, we can just determine the possible valid region of $c_{0}$ as shown in Figure 6 . However, usually the $c_{0}$-curves cannot tell us which value of $c_{0}$ gives the fastest convergent series.

To overcome this shortcoming, we determined the possible optimal value of $c_{0}$ by the minimum of averaged residual

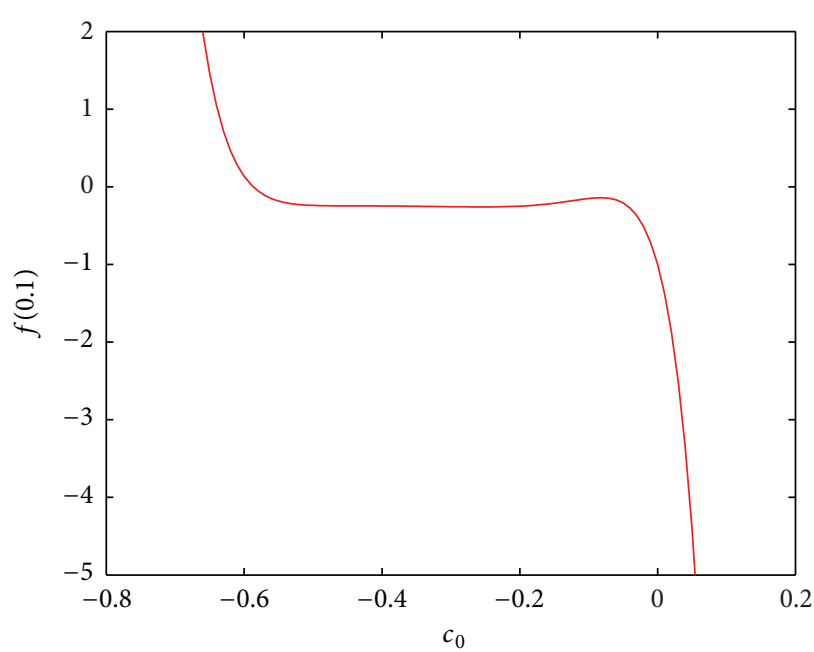

FIGURE 6: The $c_{0}$-curve of the 9th-order approximation in case of $c_{1}=c_{2}=0$.

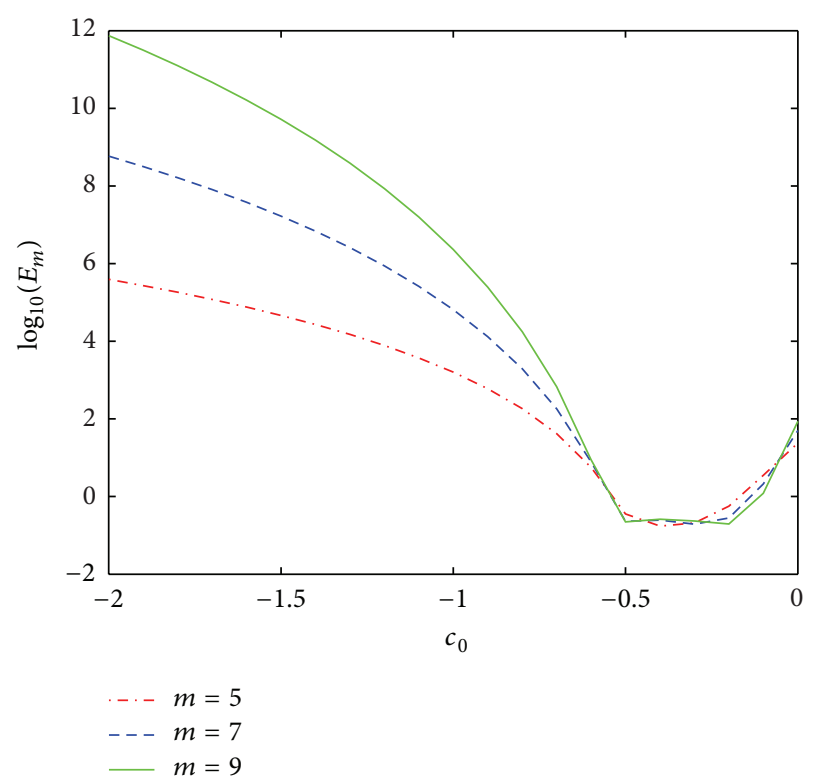

FigURE 7: Averaged residual error $E_{m}$ versus $c_{0}$ in case of $c_{1}=c_{2}=0$.

error $E_{9}$, corresponding to the nonlinear algebraic equation $E_{9}^{\prime}=0$. According to Table 6 , the value minimum of $E_{9}$ is obtained at $c_{0}=-0.44032$. The curves of $E_{m}$ versus $c_{0}$ at different order of approximation $m=5,7$, and 9 are shown in Figure 7.

4.2.2. Optimal $c_{1}=c_{2}$ in Case of $c_{0}=-1$. In this case, $E_{9}$ has the value minimum of $8.2039 \times 10^{-2}$ at the optimal point $c_{1}=c_{2}=0.0228$. Our calculations showed that the corresponding $E_{9}$ in case of $c_{0}=-1, c_{1}=c_{2}=0$ has the minimum 21.865. This illustrates that the second one-parameter optimal homotopy-analysis approach is not obviously better than the first one mentioned in Section 4.2.1. But this approach can give much better approximation than 
TABLE 7: The absolute error of approximate solution and exact solution of $f(t)$ by 15th-order approximate.

\begin{tabular}{lcccc}
\hline$t$ & $c_{0}=-0.44032, c_{1}=c_{2}=0$ & $c_{0}=-1, c_{1}=c_{2}=0$ & $c_{0}=-0.6084, c_{1}=c_{2}=0.1611$ & $c_{0}=-0.1836, c_{1}=-0.2070$, and $c_{2}=-0.4554$ \\
\hline 0 & $1.6173 e-009$ & $5.0401 e+005$ & $6.2742 e-002$ & $3.6240 e-001$ \\
0.2 & $1.2106 e-010$ & $1.6493 e+004$ & $2.0901 e-003$ & $1.1136 e-002$ \\
0.4 & $1.1105 e-010$ & $1.9738 e+002$ & $9.0034 e-005$ & $2.1428 e-002$ \\
0.6 & $1.0013 e-010$ & $3.7288 e-001$ & $1.9358 e-005$ & $1.7872 e-002$ \\
0.8 & $1.7154 e-011$ & $7.4725 e-006$ & $6.2182 e-007$ & $9.5152 e-007$ \\
1.0 & $1.3831 e-012$ & $1.6595 e-007$ & $2.0572 e-011$ & $3.4417 e-015$ \\
\hline
\end{tabular}

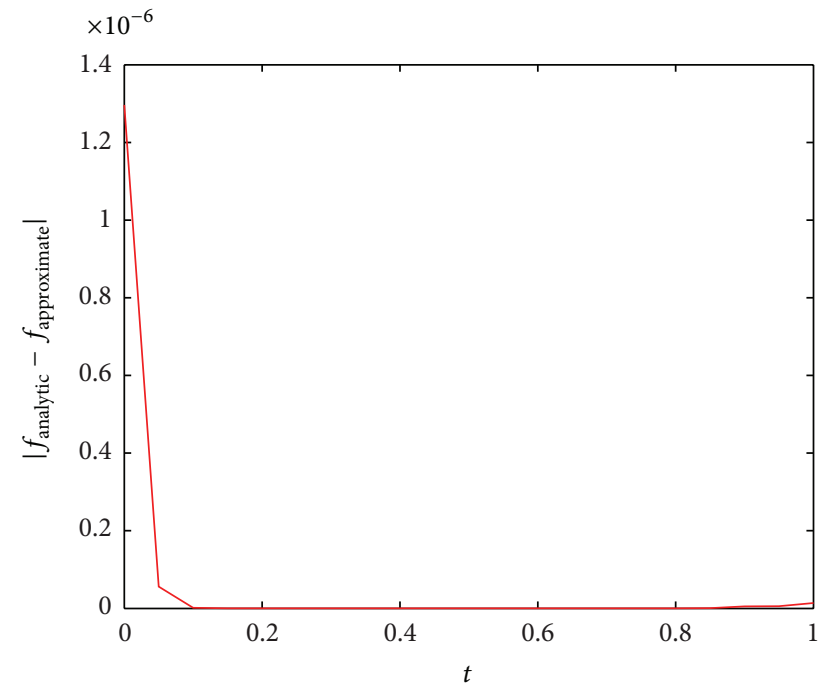

FIGURE 8: $\left|f_{\text {analytic }}-f_{\text {approximate }}\right|$ with $c_{0}=-0.44032$ and $c_{1}=c_{2}=0$.

those without optimal convergence-control parameters in case of $c_{0}=-1$ and $c_{1}=c_{2}=0$.

4.2.3. Optimal $c_{0}$ in Case of $c_{1}=c_{2}$. In this case, we obtain the unknown convergence-control parameters $c_{0}$ and $c_{1}$ in case of $c_{2}=c_{1}$. The corresponding residual error $E_{9}$ is now a function of both $c_{0}$ and $c_{1}$, which has the value minimum of $4.6895 \times$ $10^{-4}$ at the optimal point $c_{0}=-0.6084, c_{1}=c_{2}=0.1611$.

4.2.4. Optimal $c_{0}, c_{1}$, and $c_{2}$ in Case of $c_{1} \neq c_{2}$. Let us consider the optimal approach with the three unknown convergencecontrol parameters. The corresponding $E_{9}$ is now a function of $c_{0}, c_{1}$, and $c_{2}$, which has the minimum $4.94 \times 10^{-3}$ at the optimal values $c_{0}=-0.1836, c_{1}=-0.2070$, and $c_{2}=-0.4554$.

Based on the above calculations for this equation, we found that all optimal homotopy-analysis approaches can give much better approximations, which converge much faster than those without optimal convergence-control parameters in case of $c_{0}=-1$ and $c_{1}=c_{2}=0$. But in this example, the first one-parameter optimal homotopy-analysis approach is better than other cases.

The absolute error and comparison of the 15th-order approximate solution and analytic solution (49) with $c_{0}=-0.44032, c_{1}=c_{2}=0$ are shown in Figures 8 and 9, respectively. The absolute errors $\left|f_{\text {analytic }}-f_{\text {approximate }}\right|$ have been calculated for different cases of $c_{0}, c_{1}$, and $c_{2}$ in Table 7 .

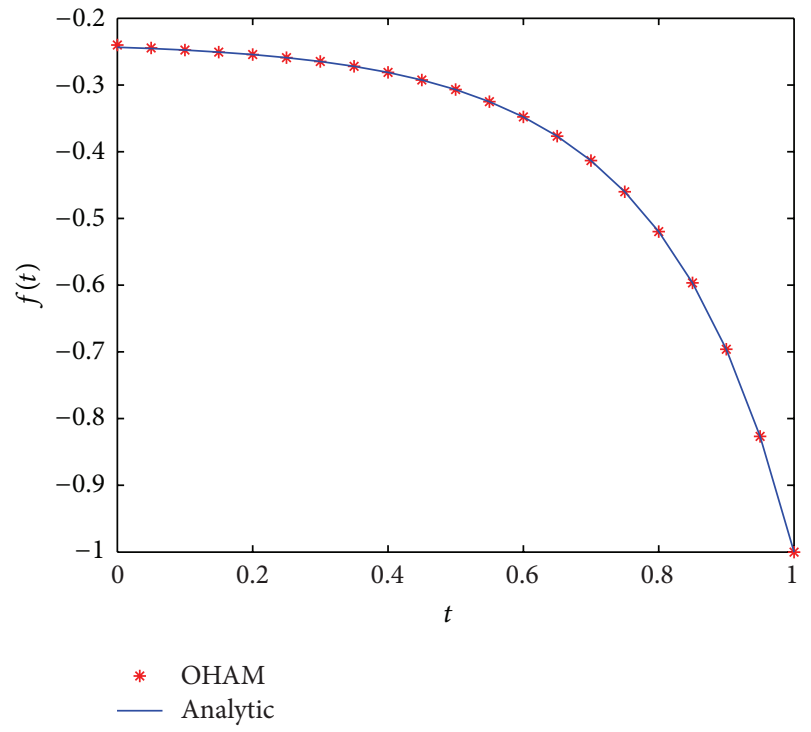

FIGURE 9: Comparison of the exact solution with the approximate solution of $f(t)$ by $c_{0}=-0.44032$ and $c_{1}=c_{2}=0$.

\section{Conclusion}

In this paper, we have successfully developed an optimal homotopy-analysis approach for solving HJB equation arising in nonlinear optimal control problems. With the deformation functions defined, the method used here contains three auxiliary convergence-control parameters, which provide us with a simple way to adjust and control the convergence region of the solution. By minimizing the averaged square residual error, the optimal convergence-control parameters $c_{0}, c_{1}$, and $c_{2}$ can be obtained.

\section{References}

[1] B. D. Craven, Control and Optimization, Chapman \& Hall, London, UK, 1995.

[2] S. Effati and H. Saberi Nik, "Solving a class of linear and nonlinear optimal control problems by homotopy perturbation method," IMA Journal of Mathematical Control and Information, vol. 28, no. 4, pp. 539-553, 2011.

[3] K. L. Teo, C. J. Goh, and K. H. Wong, A Unified Computational Approach to Optimal Control Problems, Longman Scientific \& Technical, Essex, UK, 1991.

[4] R. Bellman, Dynamic Programming, Princeton University Press, Princeton, NJ, USA, 1957. 
[5] R. W. Beard, G. N. Saridis, and J. T. Wen, "Approximate solutions to the time-invariant Hamilton-Jacobi-Bellman equation," Journal of Optimization Theory and Applications, vol. 96, no. 3, pp. 589-626, 1998.

[6] J. E. Rubio, Control and Optimization, The Linear Treatment of Nonlinear Problems, Manchester University Press, Manchester, UK, 1986.

[7] A. Fakharian, M. T. Hamidi Beheshti, and A. Davari, "Solving the Hamilton-Jacobi-Bellman equation using Adomian decomposition method," International Journal of Computer Mathematics, vol. 87, no. 12, pp. 2769-2785, 2010.

[8] H. Saberi Nik, S. Effati, and M. Shirazian, "An approximateanalytical solution for the Hamilton-Jacobi-Bellman equation via homotopy perturbation method," Applied Mathematical Modelling, vol. 36, no. 11, pp. 5614-5623, 2012.

[9] S. Effati, H. S. Nik, and M. Shirazian, "An improvement to the homotopy perturbation method for solving the Hamilton Jacobi Bellman equation," IMA Journal of Mathematical Control and Information, 2013.

[10] H. Saberi Nik, S. Effati, and A. Yildirim, "Solution of linear optimal control systems by differential transform method," Neural Computing and Applications, 2012.

[11] S. J. Liao, "A kind of approximate solution technique which does not depend upon small parameters. II. An application in fluid mechanics," International Journal of Non-Linear Mechanics, vol. 32, no. 5, pp. 815-822, 1997.

[12] S. J. Liao, "Comparison between the homotopy analysis method and homotopy perturbation method," Applied Mathematics and Computation, vol. 169, no. 2, pp. 1186-1194, 2005.

[13] S. J. Liao, "Homotopy analysis method: a new analytical technique for nonlinear problems," Communications in Nonlinear Science and Numerical Simulation, vol. 2, no. 2, pp. 95-100, 1997.

[14] S. J. Liao, Beyond Perturbation: Introduction to the Homotopy Analysis Method, CRC Press; Chapman \& Hall, Boca Raton, Fla, USA, 2003.

[15] T. Hayat, T. Javed, and M. Sajid, "Analytic solution for rotating flow and heat transfer analysis of a third-grade fluid," Acta Mechanica, vol. 191, no. 3-4, pp. 219-229, 2007.

[16] S. Abbasbandy and F. S. Zakaria, "Soliton solutions for the fifth-order KdV equation with the homotopy analysis method," Nonlinear Dynamics, vol. 51, no. 1-2, pp. 83-87, 2008.

[17] S.-P. Zhu, "An exact and explicit solution for the valuation of American put options," Quantitative Finance, vol. 6, no. 3, pp. 229-242, 2006.

[18] S. J. Liao, "An optimal homotopy-analysis approach for strongly nonlinear differential equations," Communications in Nonlinear Science and Numerical Simulation, vol. 15, no. 8, pp. 2003-2016, 2010.

[19] S. J. Liao, Homotopy Analysis Method in Nonlinear Differential Equations, Springer, Berlin, Germany, 2012.

[20] W. H. Fleming and R. W. Rishel, Deterministic and Stochastic Optimal Control, Springer, New York, NY, USA, 1975.

[21] M. R. Caputo, Foundations of Dynamic Economic Analysis: Optimal Control Theory and Applications, Cambridge University Press, Cambridge, UK, 2005. 


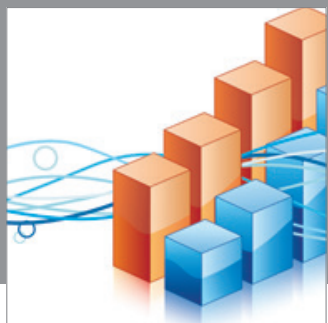

Advances in

Operations Research

mansans

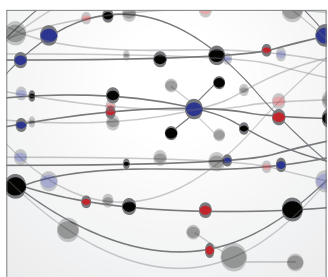

The Scientific World Journal
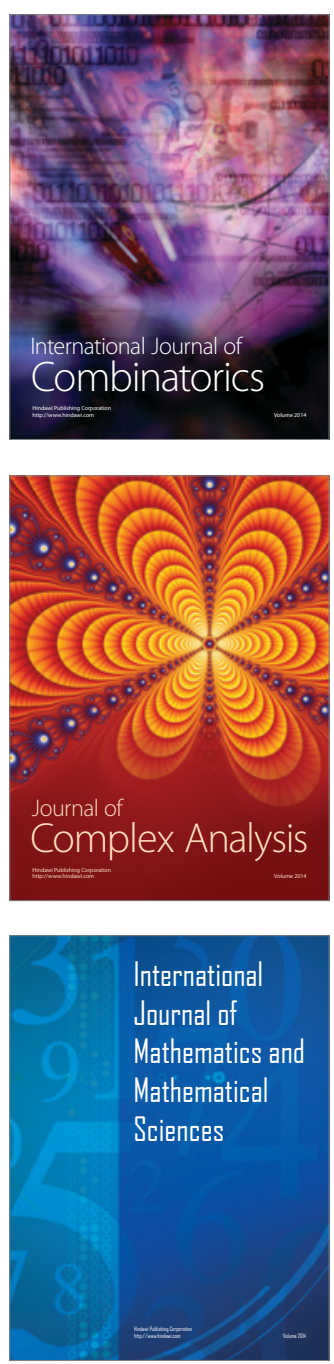
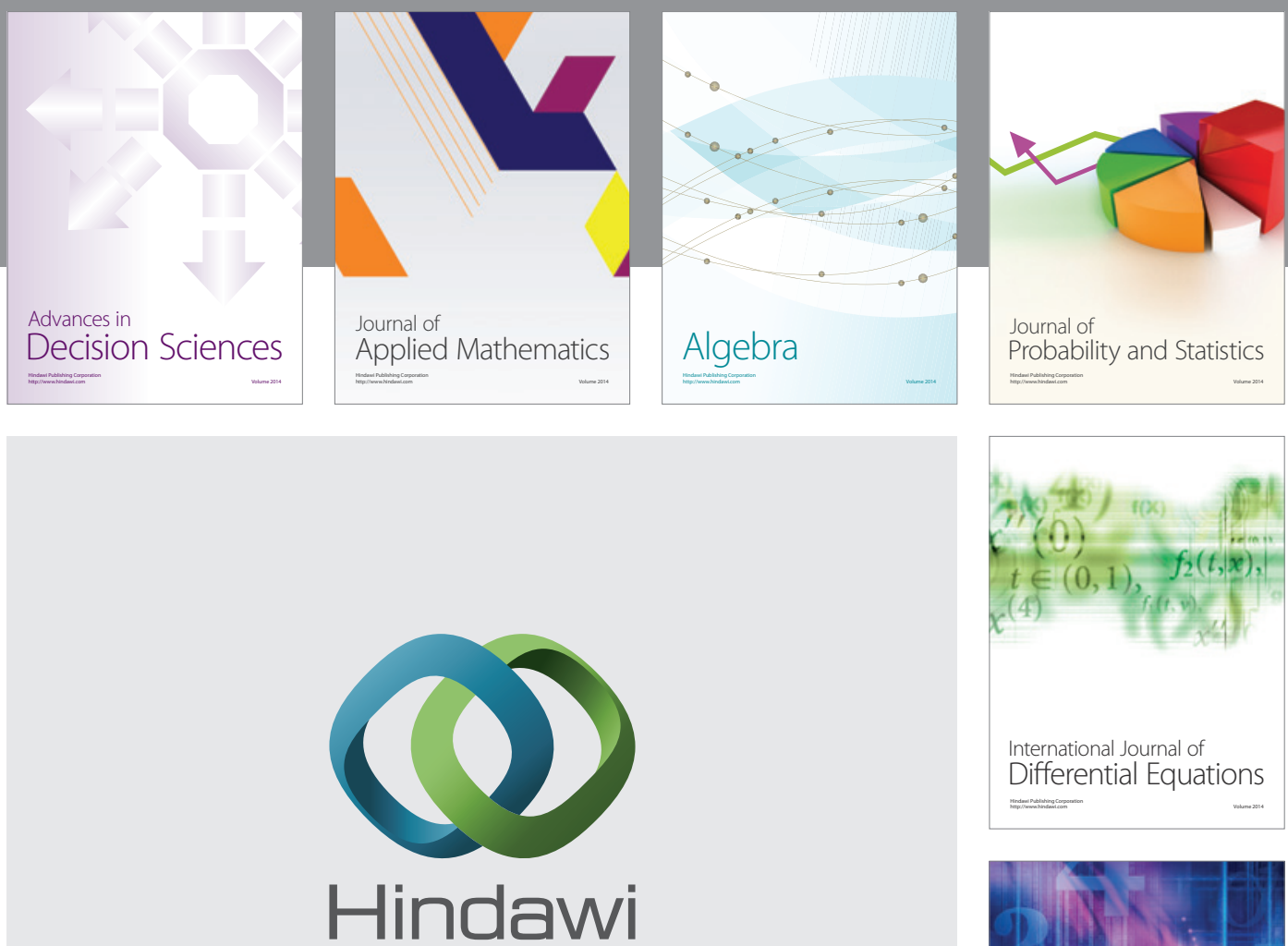

Submit your manuscripts at http://www.hindawi.com
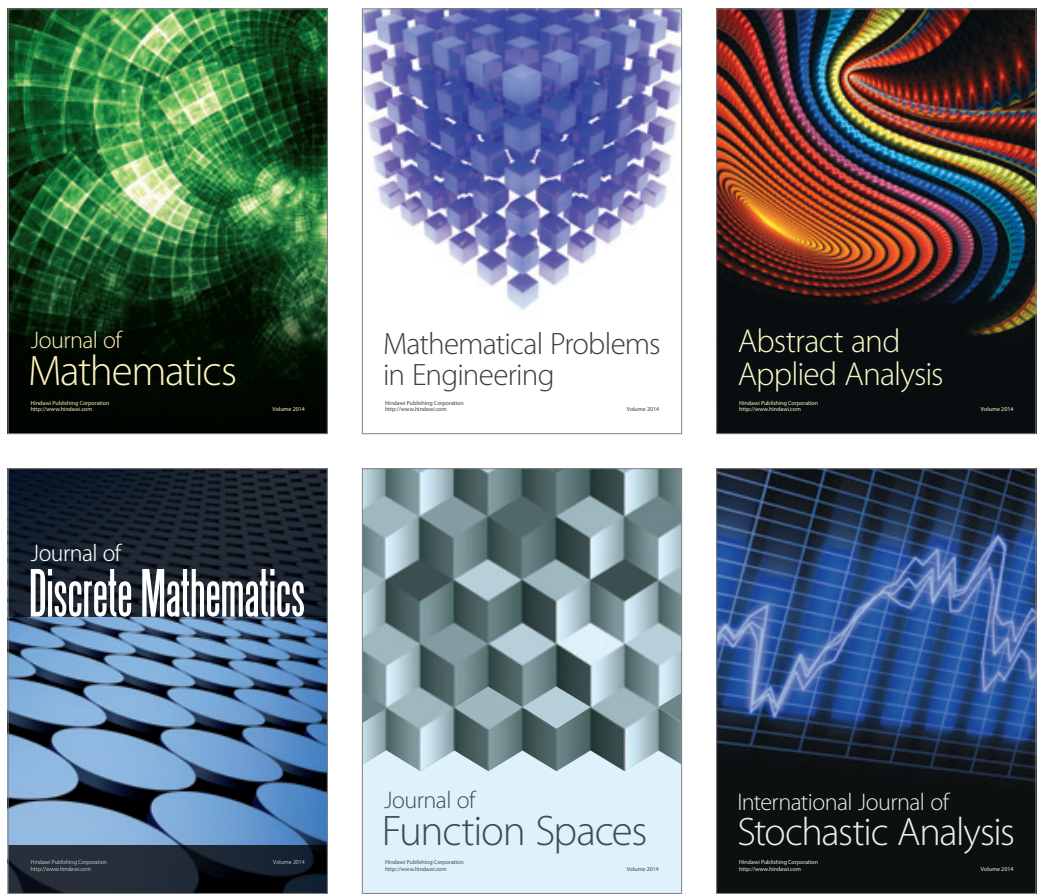

Journal of

Function Spaces

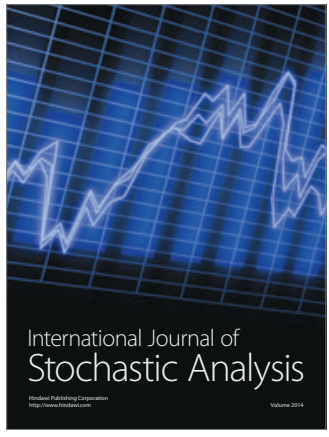

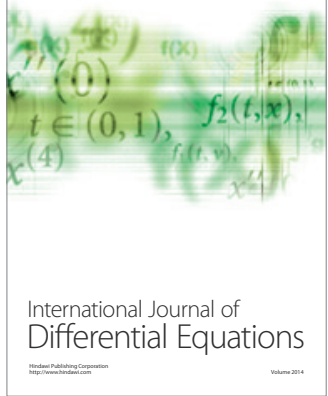
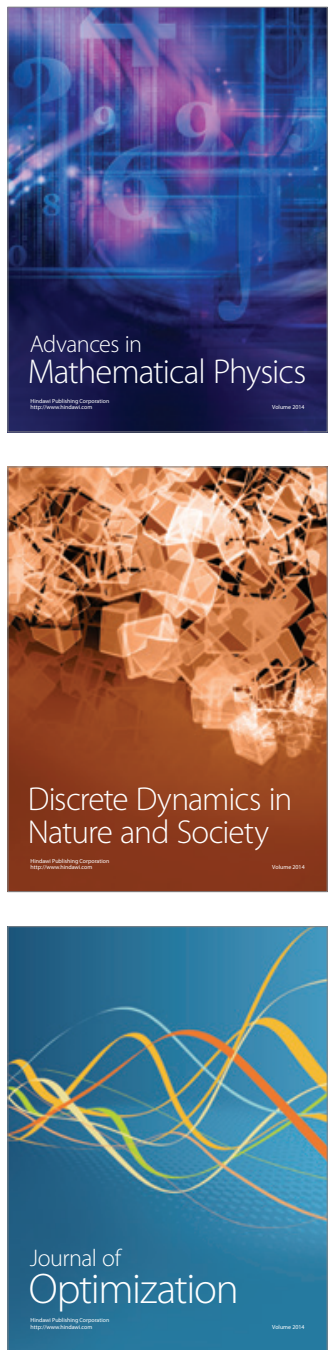